\title{
Developing Highly-Integrated Subcutaneous Biochips for Remote Monitoring of Human Metabolism
}

\author{
Sandro Carrara*, Andrea Cavallini, Sara Ghoreishizadeh, Jacopo Olivo, Giovanni De Micheli \\ EPFL - École Polytechnique Fédérale de Lausanne, Switzerland \\ *sandro.carrara@epfl.ch
}

\begin{abstract}
A highly integrated system for remote monitoring of several human metabolites is proposed. It is obtained by integrating several components, such as: bio-probes, carbon nanotubes, micro-fabricated gold-electrodes, temperature and pH sensors, extremely small CMOS IC and multilayer coil for remote powering data acquisition and transmission. The entire system has final sizes of only $2.2 \mathrm{~mm}$ in width and thick and it is long $1.5 \mathrm{~cm}$. In this paper, results on sensors micro-fabrication, sensing tests, IC design, and power transmission are presented. These results confirmed that the proposed approach is suitable for minimally invasive monitoring of multi-metabolites in humans
\end{abstract}

\section{INTRODUCTION}

Continuous monitoring of humans is already in the market for glucose [1] as well as for lactate [2] thanks to electrochemical sensing. The reliability of this technology for glucose remote monitoring has been validated up to 8 month in mice [3] and up to one year in pigs [4]. The next step will be the extension of the technology to other relevant metabolites like, for example, glutamate [5], ATP [6], and relevant drugs compounds [7]. Carbon Nanotubes have been demonstrated to enhance the sensitivity for both exogenous [7] and endogenous [8] metabolites toward physiological concentration in human blood. One of the next challenges is now to integrate all these metabolites in a highly-integrated single-subcutaneous nano-bio-chip in order to provide minimally invasive human telemetry. We need to provide extremely small chip sizes (close to those of large surgery needles) to be minimal invasive. At the same time, we need to control both temperature and $\mathrm{pH}$ [9] to provide reliability in electrochemical sensing. Last but not least, we need to also have a fully-on-board-generation of voltage ramps [8] as well as battery-less systems to contain the device size. Voltage ramps may be generated in chip with smart solutions [10] while battery-less implant is address by remote powering [11].

The aim of the present paper is to propose the first preliminary tests in developing a complete system that addresses this challenge

\section{SYSTEM LEVEL DESIGN}

At system level, the whole device is obtained by integrating three main parts (Fig. 1): a passive chip realized by metal evaporation and oxide formation onto silicon chip with size in $2.2 \mathrm{~mm} \times 1.5 \mathrm{~cm}$; an integrated CMOS circuit implemented in $0.18 \mu \mathrm{m}$ technology that has dimensions $1.522 \mathrm{~mm} \times 1.522$ $\mathrm{mm}$; a multi-coil with a thickness no larger than $816 \mu \mathrm{m}$ for receiving power by inductive link. The passive chip has been fabricated on silicon wafers pre-treated with $500 \mathrm{~nm}$ of silicon oxide. Platinum has been chosen for the whole metallization; the on-chip sensors have been passivated with an atomic layer deposition of $20 \mathrm{~nm}$ of $\mathrm{Al}_{2} \mathrm{O}_{3}$. The CMOS IC includes both the readout circuit and the ramp generator. The ramp generator is realized using a semi custom design from digital and analog blocks, the digital blocks are implemented using Mentor graphics and encounter for place and routing and the analog is implemented using Cadence virtuoso. The multi-layer receiving coil has been obtained by spiral inductors replicated on the two layers of different printed circuit boards and, then, electrically connected. The three parts have been realized and tested separately.

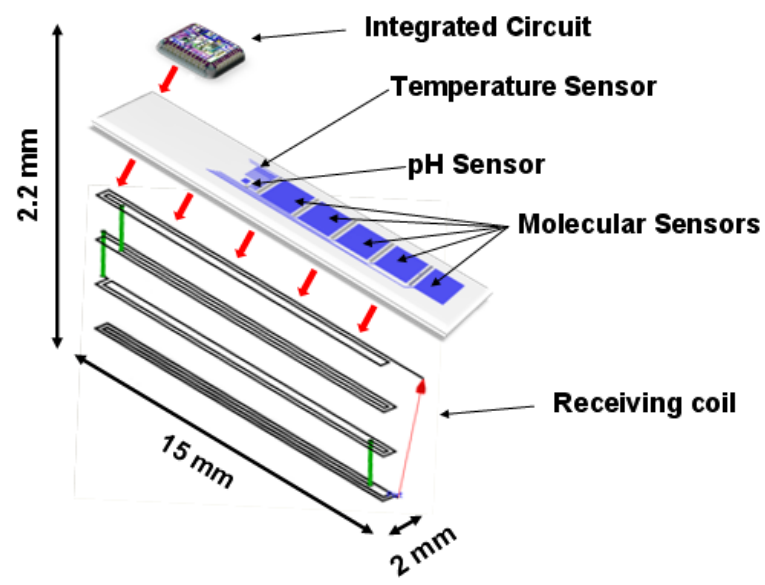

Figure 1 System-level design 


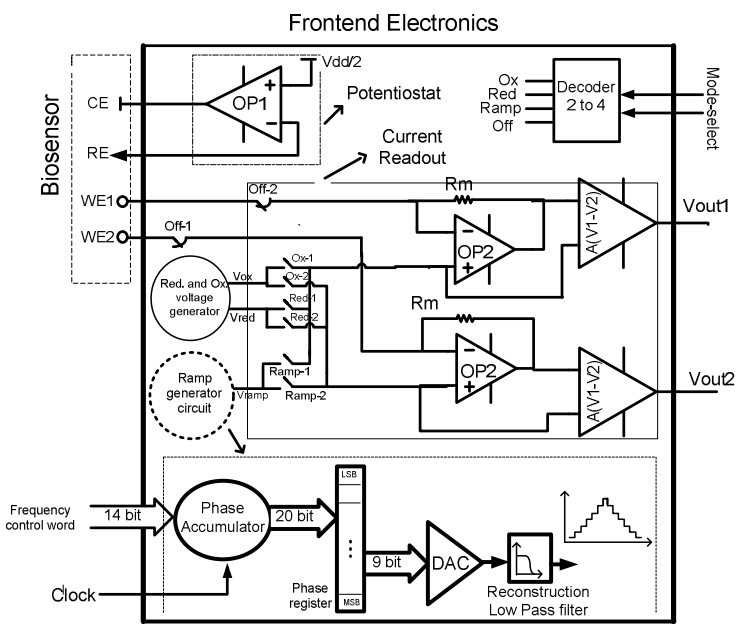

Figure 2 Proposed circuit architecture for the biosensing frontend electronics

A. Passive Chip

\section{BUILDING-BLOCKS DESIGN}

The sensing platform has been fabricated in the Center of MicroNano Technology of the EPFL. The chip measures 2.2 x $15 \mathrm{~mm}$, and hosts a counter and a reference electrode, 5 independent working electrodes (diameter $500 \mu \mathrm{m}$, area $196250 \mu \mathrm{m}^{2}$ ), a platinum resistive thermal device for temperature monitoring, a $\mathrm{pH}$ sensor and a slot for socketing the measuring circuit. The sensing platform has been fabricated on silicon wafers pre-treated with $500 \mathrm{~nm}$ of silicon oxide. Platinum has been chosen for the whole metallization; the sensor has been passivated with an atomic layer deposition of $20 \mathrm{~nm}$ of $\mathrm{Al}_{2} \mathrm{O}_{3}$.

Chitosan solution $0.7 \% \mathrm{w} / \mathrm{v}$ has been made by dissolving chitosan flakes (medium molecular weight - Aldrich) in a solution of acetic acid $2 \% \mathrm{pH} 3$. The solution has been stirred for several hours until complete dissolution, filtered to remove the un-dissolved particles and sonicated 2 hours to improve its fluidity. Final $\mathrm{pH}$ was then set to 5. MWCNT (diameter $10 \mathrm{~nm}$, lengths $1-2 \mu \mathrm{M}, \mathrm{COOH}$ content $5 \%$ Dropsens) were finally added to the CNT solution at the concentration of $10 \mathrm{mg} / \mathrm{ml}$. The solution was then sonicated again for 2 hours until complete dissolution of the CNT aggregates. Electrochemical measurement and electrodepositon have been performed by using an Autolab potentiostat (Ecochimie). Electrodeposition has been done by covering the electrochemical cell of the sensing platform with the chitosan-CNT solution, and applying a constant potential of $+1.5 \mathrm{~V}$ for 100,300 or 600 ". The chips were then rinsed with milliq water to remove the solution in excess. Chronoamperometry has been performed in a $1 \mathrm{x}$ PBS solution $\mathrm{pH} 7.4$ under mild stirring conditions. The electrodes were first conditioned for 1200 " at $+900 \mathrm{mV}$, then tested with injections of $\mathrm{H}_{2} \mathrm{O}_{2} 10 \mathrm{mM}$ at $+650 \mathrm{mV}$.

\section{B. Active Chip}

To be able to miniaturize and integrate the sensor and the electronics into a single chip to realize an implantable biosensor platform, the required front-end electronics for the realization of two mostly used detection techniques, ChronoAmperometry (CA) and Cyclic Voltammetry (CV), must be carefully designed. The literature is limited regarding the integration of the waveform generator to the implantable biochip that is essential for fully integrated CV method. Most of the designed electronics for the biosensors use external generator [12], [13]. Reference [14] introduces an amperometric instrumentation system for biosensor application with an on chip ramp generator circuit. It has been designed for single target detection as all the Working Electrodes (WE) arrays has the same potential. The main difference of our work is that here the sensors are sharing the Counter (CE) and Reference (RE) electrodes within a chamber, and each WE presents an individual sensor, though they may require different potentials or even different methods to be activated. So the voltage (either the ramp voltage or the fixed potential) must be applied somehow to WE instead of RE, to allow the sensor array to sense different compounds properly. Fig. 2 shows the architecture of the proposed frontend electronics. It consists of three main parts: i) a ramp generator circuit; ii) a potentiostat; iii) a current readout circuit. The ramp generator circuit generates a verylow slope and low-frequency ramp voltage. The circuit is based on the Direct Digital Synthesis (DDS) method. A DDS waveform generator includes a numerically controlled oscillator and a Digital-to-Analog Converter (DAC). The former produces a discrete-time, quantized version of the desired output waveform whose period is controlled by the digital word contained in the Frequency-Control-Register (FCR). The sampled, digital waveform is converted to an analog waveform by the DAC. The ramp generator circuit generates a triangular waveform with a controllable slope that varies from less than $10 \mathrm{mV} / \mathrm{sec}$ to more than $100 \mathrm{mV} / \mathrm{sec}$ with a rail-to-rail swing. The steps are $3.3 \mathrm{mV}$ and the size of the circuit is $0.122 \mathrm{~mm}^{2}$ [15]. The potentiostat is realized with only one operational amplifier OP1 to reduce the power dissipation and the induced noise by electronics to the measured current.

The readout circuit senses currents in the range of $+/-5 \mathrm{uA}$ with an equivalent input referred current noise of $3 \mathrm{nA} \mathrm{rms}$ and translates it into a voltage. The amplification is achieved in two stages with OP2 and its next stage. OP2 is a folded cascade amplifier with a dual n- and p-MOS input stage and a class A output stage. The dual stage input structure is used to ensure rail-to-rail input voltage. The next stage is an analog subtractor and amplifier circuit with a gain of 10 . This stage has been added to amplify the net voltage across the resistor [16].

\section{Receiving Coil}

Remote powering through inductive link is one of the most promising approaches to provide power to implantable sensors. The miniaturization of the implanted inductors, while preserving the power efficiency, is still an open topic. 


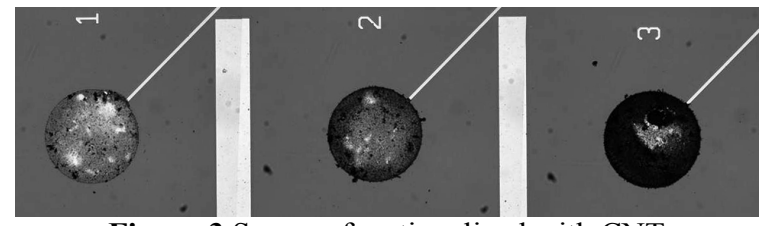

Figure 3 Sensors functionalized with CNT

To this end, a multi-layer approach is studied for the implantable coil. Multi-layer approach has been widely studied for integrated circuits $[17,18]$. However, it is not commonly used on printed circuit boards for inductive powering. In a multi-layer coil, a spiral inductor is replicated on the two layers of different printed circuit boards; then, the different boards are stacked and the inductors are electrically connected, as shown in Fig.1. Due to the higher number of turns, a multi-layer inductor has a higher coupling with the external inductor, when compared to a single-layer inductor having the same area. A detailed study of a multi-layer approach is reported in [19], together with a comparison with a single-layer approach.

The system also performs bidirectional data communication. Downlink communication (from the external part to the implanted device) is obtained by means of Amplitude Shift Keying (ASK). Uplink communication (from the internal device to the external part) is obtained by using backscattering technique. In such a technique, the internal load is changed accordingly to outgoing bit-stream. That change is detected by the external part as a change on the current flowing on the external inductor. Thus, bidirectional data communication is performed without any implanted RF transmitter.

\section{A. Passive Chip}

Fig. 3 shows 3 working electrodes of the same chip coated with chitosan and carbon nanotubes for different electrodeposition times: pad $1-100$ " electrodeposition, pad 2 $-300 "$, pad $3-600 "$.

\section{TESTS ON THE BUILDING BLOCK}

As can be seen, the film formation is located only above the working electrode area (Fig. 3), while there is no significant trace of CHT-CNT solution above the counter electrode in the rest of the chip. The amount of CNT entrapped increases with the electrodeposition time (Fig. 3). Higher times grant a denser coverage of the electrode surface. For all the 3 electrodes, there is a clear region of higher density in the proximity of the electrode edges. This is probably due to the electric field applied for the electrodeposition, which is planar, and not perpendicular to the working electrodes. Fig. 4 shows the chronoamperometries at $+650 \mathrm{mV}$ versus $\mathrm{H}_{2} \mathrm{O}_{2}$ for each electrodeposition time. Error bars represent the instrument noise. Each point of the series has been obtained by subtracting the current in absence of $\mathrm{H}_{2} \mathrm{O}_{2}$ with the current obtained after each injection.

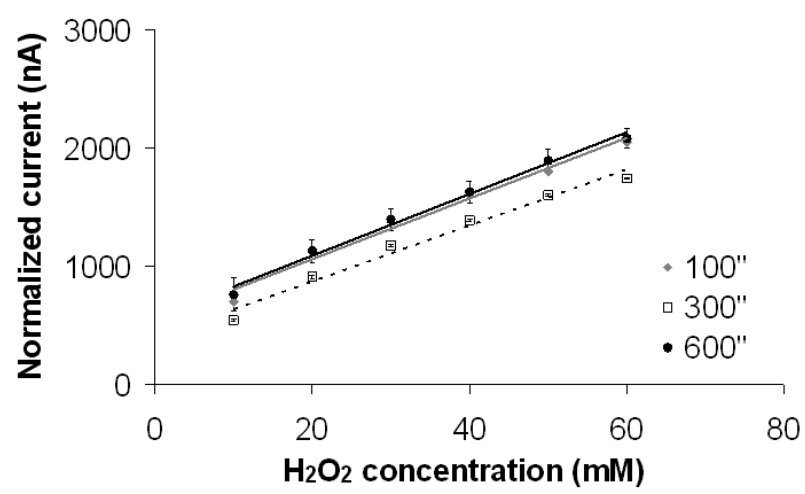

Figure 4 Different sensitivities for different depositions

Sensitivities in $\mathrm{pA} / \mathrm{mM} \mathrm{u \textrm {m } ^ { 2 }}$ are respectively $0.13,0.12$ and 0.13 for the electrodeposition times of 100,300 and 600 ". These results show that increasing the deposition time, and therefore increasing CNT density and film thickness, does not improve the response signal for the considered times. Moreover, increasing error bars for the deposition time of 600 " indicate that thicker or denser films decrease the electrode performance due to their high noise.

Chitosan electrodeposition proved to be capable to create complex nanostructuration in a controlled fashion. The addition of enzymes like cytochromes P450 or glucose oxidase to the chitosan-CNT solution represent an interesting perspective for the single-step creation of biosensors in a cheap, quick and reproducible way, for any kind of electrode geometry and dimension

\section{B. Active Chip}

The circuit has been implemented in $0.18 \mu \mathrm{m}$ technology. It has been simulated in Cadence Virtuoso using the electrical equivalent model of the biosensor from [20]. Simulation results indicate highly controllable and low slope triangular waveforms, and also highly linear current to voltage transformation in the readout circuit [15.16].

The power consumption of the proposed interface electronics, including the potentiostat, readout circuit, ramp generator, and the reference voltage generators is $530 \mu \mathrm{W}$ in the worst case, which is suitable for remotely powering the implantable biosensor. Fig. 5 shows the electrode and the chip that is placed and fixed on top of the electrode and is ready to be tested.

\section{Receiving coil}

A high efficiency class-E power amplifier was utilized to drive the external inductor. Powered by two thin lithium-ion polymer batteries, the system can transfer up to $15 \mathrm{~mW}$ over a distance of $6 \mathrm{~mm}$ in air. The receiving coil was obtained by using an 12-layers, 21-turns receiving inductor having an area of $30 \mathrm{~mm}^{2}$ and a thickness of $816 \mu \mathrm{m}$ (Fig. 6). [19]. The maximum link efficiency measured is $13 \%$. Furthermore, the system can transfer up to $1.17 \mathrm{~mW}$ when a $17 \mathrm{~mm}$ beef sirloin is placed between the inductors. 


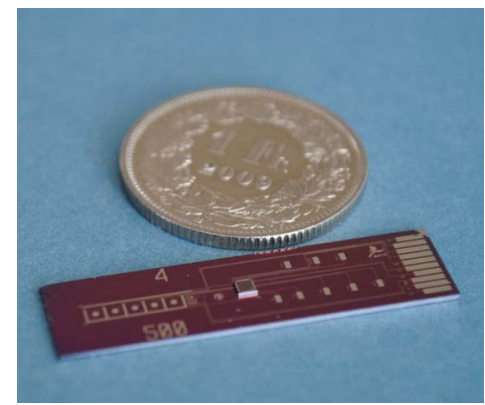

Figure 5 The realized passive and active chips

The multi-layer approach can partially compensate an area reduction by accordingly increasing the number of layers. The smaller size can noticeably simplify the implantation and decreases the discomfort of the patient.

The system using the multi-coils receiving antenna was able to perform downlink communication with a bit-rate of $100 \mathrm{kbps}$. Uplink communication is performed up to $66.6 \mathrm{kbps}$, over a maximum distance of $1.1 \mathrm{~cm}$.

\section{CONCLUSION}

In this paper a highly integrated system for human metabolism telemetry is presented. The three main part of the system have been realized: the passive chip has on-board the molecular, the temperature, and the $\mathrm{pH}$ sensors; the CMOS integrated circuit that provide the reading of the sensors; the multi-layer antenna for receiving power and transmitting sensing data. The passive chip has been functionalized with proper bio and nanostructures and it returns sufficiently high sensitivity for future measurements including oxidases. The CMOS IC showed good performance in simulations. The receiving antenna collected enough energy for supporting the work of the IC. All these preliminary tests demonstrate the feasibility of the entire system. Future works will be the integration of the three sub-systems and the test on the performance of the fully embedded system.

\section{Acknowledgments}

This research has been supported by different sources of founding: the i-IronIC project, financed with a grant from the Swiss Nano-Tera.ch initiative and evaluated by the Swiss National Science Foundation. The research has also been supported by the projects Sinergia CRSII2_127547/1

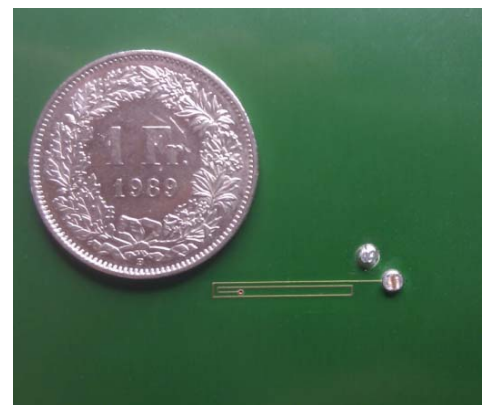

Figure 6 Top layer of the realized receiving inductor

\section{References}

[1] F. Valgimigli, F. Lucarelli, C. Scuffi, S. Morandi, I. Sposato, Journal of Diabetes Science and Technology, 4(2010) 1182-1192

[2] A. Poscia, D. Messeri, D. Moscone, F. Ricci, F. Valgimigli, , Biosensors and Bioelectronics 20 (2005) 2244-2250

[3] B. Yu, N. Long, Y. Moussy, F. Moussy, Biosensors and Bioelectronics 21 (2006) 2275-2282

[4] D. A. Gough, L. S. Kumosa, T. L. Routh, J. T. Lin, J. Y. Lucisano, Sci Transl Med 2(2010) 1-8

[5] S. Carrara, L. Bolomey, C. Boero, A. Cavallini, E. Meurville, G. De Micheli, F. Grassi, T. Rezzonico, Proceedings of IEEE Sensors 2011, in press.

[6] A. Cavallini, G. De Micheli, S. Carrara, Sensor Letters, 9 (2011) 18381844(7)

[7] S. Carrara, A. Cavallini, V. Erokhin, G. De Micheli, Biosensors and Bioelectronics 26 (2011) 3914-3919

[8] S. Carrara, V. V. Shumyantseva, A. I. Archakov, B. Samorì, Biosensors and Bioelectronics 24 (2008) 148-150

[9] S. Carrara, M. D. Torre, A. Cavallini, D. De Venuto, G. De Micheli, IEEE International Conference BioCAS 2010, Cyprus November 3-5, 2010, pag. 146-149

[10] H.P Forghanizadeh, G.A. Rincon-Mora, Journal of Low Power Electronics, vol.2, pp 1-5, 2006.

[11] J. Olivo, S. Carrara, G. De Micheli, IEEE Sensors Journal, 11(2011), 1573-1586

[12] M. Kimura, H. Fukushima, Y. Sagawa, K. Setsu, H. Hara, S. Inoue, IEEE Trans. Electron Devices, vol. 56, pp. 2114-2119, 2009.

[13] J.Zhang, Y.Huang, N.Trombly, C.Yang, and A.Mason, Proceedings of the IEEE Sensors Conference, Irving, CA, 2005, pp. 385-388.

[14] Lin Li; Qureshi, W.A.; Xiaowen Liu; Mason, A.J., IEEE Biomedical Circuits and Systems Conference (BioCAS), pp.294-297, 2010

[15] S. Ghoreishizadeh, C. Baj-Rossi, S. Carrara, and G. De Micheli, proceedings of IEEE/NIH 5th Life Science Systems and Applications Workshop(LiSSA), Maryland, USA, April 2011, pp. 28 -33.

[16] S. Ghoreishizadeh, S. Carrara, and G. De Micheli, IEEE Biomedical Circuits and Systems Conference (BioCAS), 2011, USA

[17] J. Xie, W. Yin, J. Shi, K. Kang, and Z. Chen, Microwave and Optical Technology Letters, vol. 49, no. 12, pp. 3180-3184, Dec. 2007.

[18] A. Zolfaghari, A. Chan, and B. Razavi, IEEE Journal of Solid-State Circuits, vol. 36, no. 4, pp. 620-628, Apr. 2001.

[19] J. Olivo, S. Carrara, and G. De Micheli, IEEE Transaction of Circuit and System for Biomedical Applications, Submitted Sept. 2011

[20] M.Ahmadiand, G.Jullien, IEEE Transactions on Circuits and Systems I: Regular Papers, vol. 56, no. 7, pp. 1339 -1348, July 2009. 